\title{
Energy Loss Estimates at Several Beam Intensities in the Fermilab Booster
}

\author{
Xi Yang and James MacLachlan \\ Fermi National Accelerator Laboratory \\ Box 500, Batavia IL 60510
}

\begin{abstract}
The difference between the effective rf voltage and the accelerating voltage required to match the rate of change of the Booster magnetic field can be used to estimate the energy loss per beam turn. Although the effective rf voltage (RFSUM) and the synchronous phase can be experimentally measured and used to calculate the accelerating voltage, the calibration of the signals during the fast change of the Booster rf frequency is difficult and appears to introduce some offset to the beam energy loss estimation. An observed linear relationship between energy loss and beam intensity is used to evaluate the offset, which is then applied to the experimental data. This approach, rather than recalibrating the signals, is simple and suitable for minimizing the error in the data.
\end{abstract}

\section{Introduction}

It is important to develop an experimental procedure to analyze the energy loss per beam turn at different times of the Booster cycle for different beam intensities. The energy loss per beam turn can be estimated from the difference between the effective accelerating voltage and the accelerating voltage required by $\mathrm{dB} / \mathrm{dt}$ alone. The measured synchronous phase $\left(\varphi_{s}\right)[1]$ and RFSUM (V) [2] are used to calculate the effective accelerating voltage $\left(\mathrm{V} \times \operatorname{Sin}\left(\varphi_{s}\right)\right)$. The rate of change of the Booster magnetic field in a cycle is used for the calculation of the accelerating voltage $\left(V_{a}\right)$ per beam turn required by $\mathrm{dB} / \mathrm{dt}$, as shown in eq. (1).[3] $\mathrm{dP} / \mathrm{dt}$ is calculated using eq. (2).

$$
\begin{aligned}
& V_{a}(t)=\left(\dot{E}(t) \times T_{\text {rev }}(t)\right) / e \\
& =\left(\beta(t) \times c \times \dot{P}(t) \times T_{\text {rev }}(t)\right) / e \\
& =((2 \pi \times R) \times \dot{P}(t)) / e \\
& \dot{P}(t)=d P / d t=\left(\left(P_{f}-P_{i}\right) / 2\right) \times \sin (2 \pi \times f \times t) \times(2 \pi \times f)
\end{aligned}
$$


$T_{\text {rev }}(t)$ is the revolution period at time $t$ in a Booster cycle, $\beta$ is the Lorentz relativistic factor, $R$ is the Booster radius, and $f$ is the Booster cycle repetition rate. $P_{f}$ is the momentum of a proton at extraction, and $P_{i}$ is the momentum of a proton at injection.

\section{Experimental Results}

RFSUM and the synchronous phase were measured at extracted beam intensities of $0.315 \times 10^{12}$ protons, $1.18 \times 10^{12}$ protons, $2.1 \times 10^{12}$ protons, $2.92 \times 10^{12}$ protons, $3.74 \times 10^{12}$ protons, $4.5 \times 10^{12}$ protons, $5.2 \times 10^{12}$ protons, and they are shown in Figs. $1(\mathrm{a})$ and $1(\mathrm{~b})$. Also, the synchronous phase at the period between injection at $\mathrm{t}=0$ and $8.5 \mathrm{~ms}$ is independent of the beam intensity, and is shown in Fig. 1(c). The black, red, green, blue, cyan, magenta, yellow curves in each plot represent the results for the several intensities from lowest to highest respectively. The accelerating voltage per beam turn required by $\mathrm{dB} / \mathrm{dt}$ using eq. (1) is shown in Fig. 1(d). The energy loss per beam turn estimated from the difference between the effective accelerating voltage and the accelerating voltage required by $\mathrm{dB} / \mathrm{dt}$ is shown in Fig. 1(e); as before the black, red, green, blue, cyan, magenta, yellow curves give the results for extracted beam intensities of $0.315 \times 10^{12}$ protons, $1.18 \times 10^{12}$ protons, $2.1 \times 10^{12}$ protons, $2.92 \times 10^{12}$ protons, $3.74 \times 10^{12}$ protons, $4.5 \times 10^{12}$ protons, $5.2 \times 10^{12}$ protons respectively.

Some error is clearly indicated by the negative energy loss in Fig. 1(e) in the period from $7 \mathrm{~ms}$ after injection to transition crossing. Such error could be generated by calibration errors in the synchrotron phase detector signal, the RFSUM signal, etc. or the alteration of the rf drive by the feedback system and the radial offset (ROF), for example.

Some investigation of the cause of the apparent negative energy loss has been carried out. First, the frequency change of the Booster rf stations was checked at the extracted beam intensity of $5.2 \times 10^{12}$ protons against calculated values; the results are shown in Fig. 2 . The red curve is calculated; the black curve is the measurement. The difference is too small to be the cause the apparent negative energy loss. Also, ROF was set to three different values, as shown by the blue curves in Fig. 3(a). The synchronous phase was recorded at those conditions, and the results are shown in Fig. 3(b). The differences among those curves are also practically negligible. 


\section{Solution}

Without recalibrating all the instruments used in the measurement, the offset to the energy loss data at different times in a Booster cycle can be obtained numerically, and applied to the data shown in Fig. 1(e). Three examples for finding the offset at $13.0 \mathrm{~ms}$, $14.0 \mathrm{~ms}$, and $23.0 \mathrm{~ms}$ after injection are shown in Figs. 4(a) - 4(c). The red lines are obtained by the linear fit of the data for the intensity vs. energy loss, and the offset is the energy loss at zero beam intensity. The linear relationship between the energy loss and the beam intensity is only observable later than $8 \mathrm{~ms}$ after injection. After the offset during the period of $8.5 \mathrm{~ms}$ after injection to extraction is applied to the energy loss shown in Fig. 1(e), the results are as shown in Fig. 4(d). The energy loss during the period that the offset couldn't be applied is shown in Fig. 4(e). The black, red, green, blue, cyan, magenta, and yellow curves are again for extracted beam intensities of $0.315 \times 10^{12}$ protons, $1.18 \times 10^{12}$ protons, $2.1 \times 10^{12}$ protons, $2.92 \times 10^{12}$ protons, $3.74 \times 10^{12}$ protons, $4.5 \times 10^{12}$ protons, and $5.2 \times 10^{12}$ protons in Fig $4(\mathrm{~d})$ and Fig. 4(e).

\section{Future Development}

For diagnostic purposes, the requirements for the RFSUM and synchrotron phase detector signals are much stricter than those for operational monitoring. They may need to be more carefully calibrated in the future or, at least, carefully checked by evaluation of independent parameters.

\section{Summary}

We have evaluated the energy lost per beam turn throughout the Booster cycle. The results have been separated into two time regions, viz., between injection at $\mathrm{t}=0$ and 8.5 $\mathrm{ms}$ and between $8.5 \mathrm{~ms}$ and extraction at about $33 \mathrm{~ms}$. In the earlier region the energy loss does not strongly depend on beam current; after $8.5 \mathrm{~ms}$ the energy loss is practically linear in beam intensity but has a pedestal which depends on time in the cycle; the pedestal has been evaluated by extrapolating the measurements to zero intensity and thereafter used to adjust the data. We do not know why the time between injection and $8.5 \mathrm{~ms}$ does not appear to have intensity-dependent loss. We do know that the local loops on the rf stations, which correct the ferrite bias current, show strong error signals which 
are very similar or identical whether there is beam present or not during this early part of the cycle. Therefore, although we do not understand the early-time observations fully, there is indication that the effects are not simply instrumental. For the present we exclude the problematic period from our analysis and conclusions.

\section{References:}

[1] X. Yang and R. D. Padilla, A Synchronous Phase Detector for the Fermilab Booster, FERMILAB-TM-2234.

[2] J. Steimel, Fermilab / Accelerator Division / RF Department / HLRF (FanBack / FanOut) Document. (1992)

[3] Edited by A. W. Chao and M. Tigner, Handbook of Accelerator Physics and Engineering. (2002) 


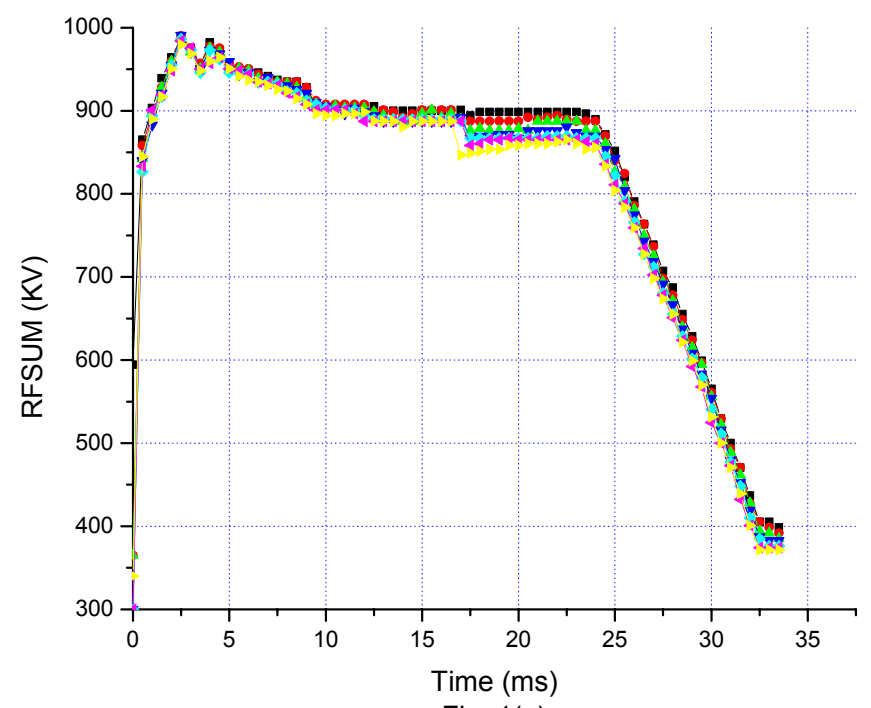

Fig. 1(a)

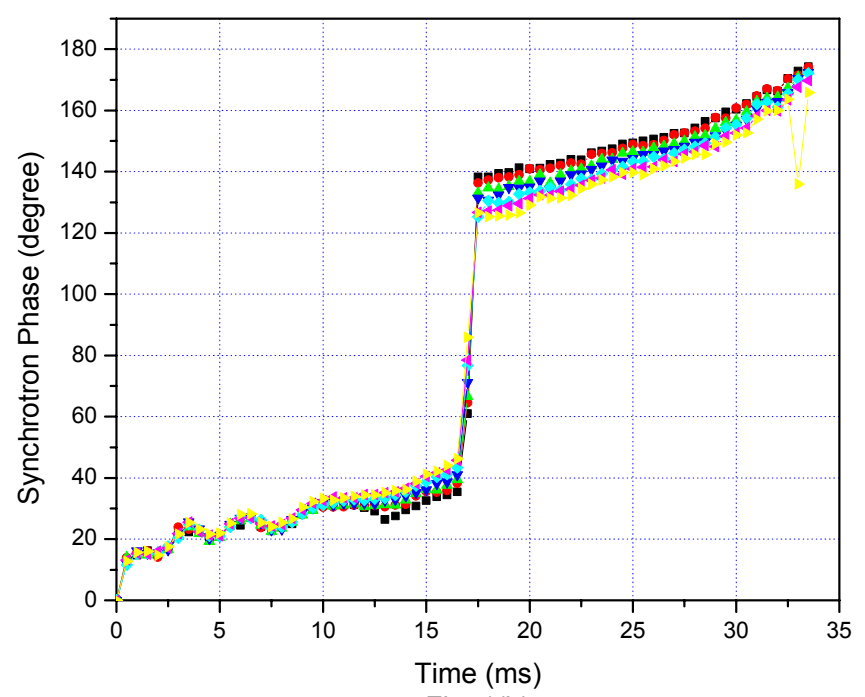

Fig. 1(b) 


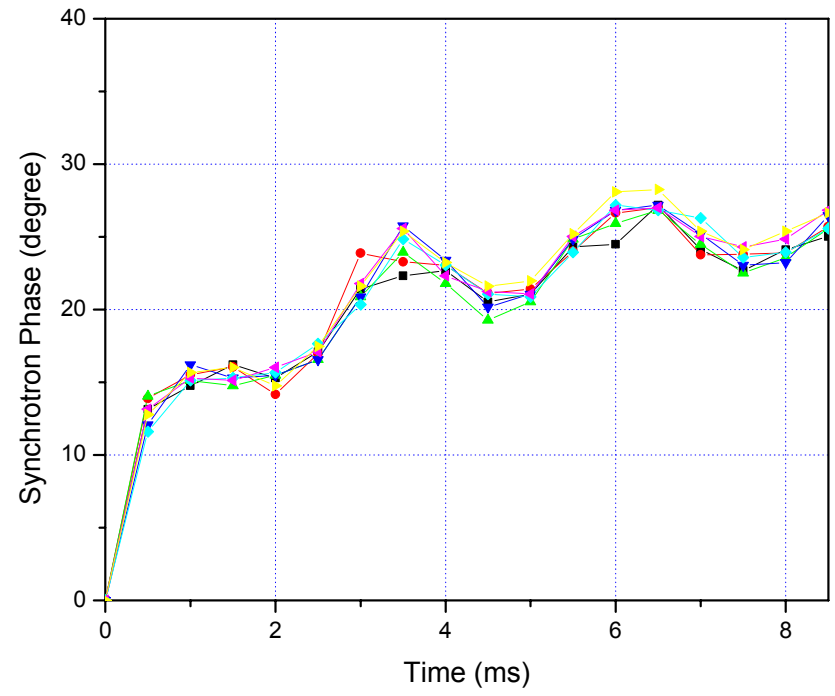

Fig. 1(c)

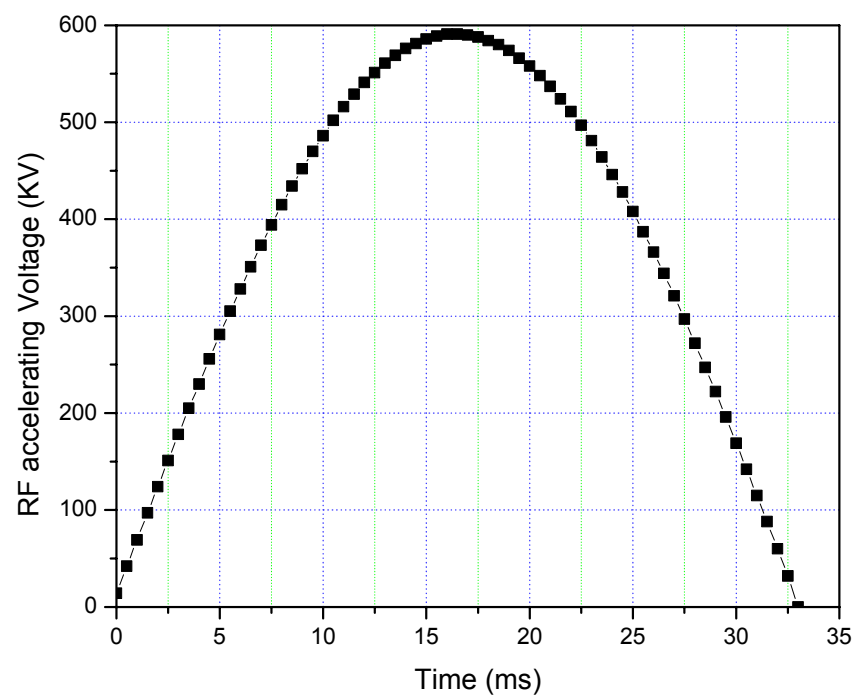

Fig. 1(d) 


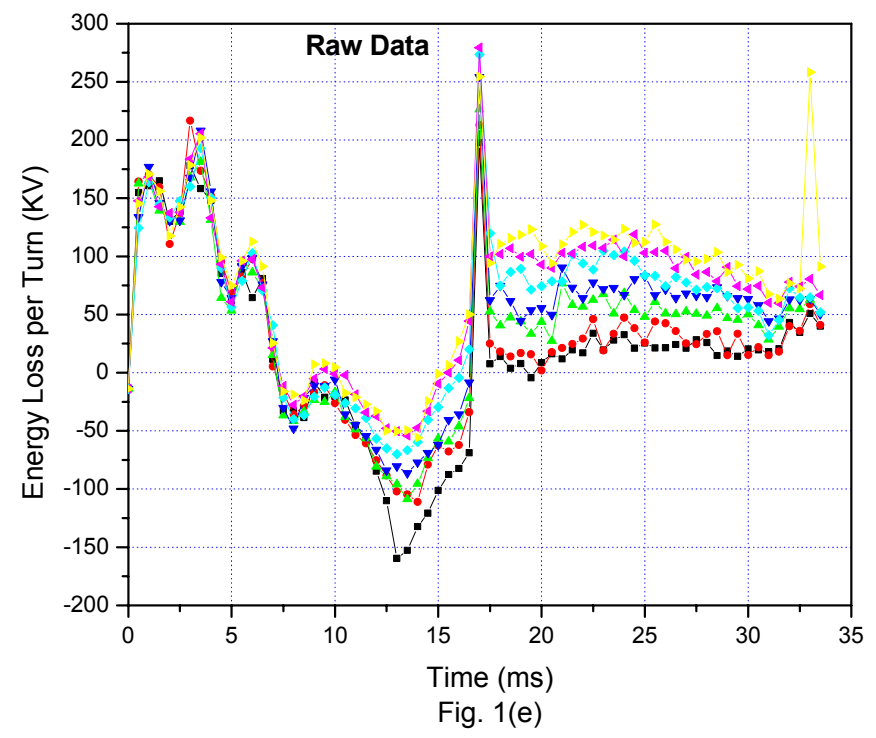

Fig. 1(a) RFSUM measured at extracted beam intensities of $0.315 \times 10^{12}$ protons (black curve), $1.18 \times 10^{12}$ protons (red curve), $2.1 \times 10^{12}$ protons (green curve), $2.92 \times 10^{12}$ protons (blue curve), $3.74 \times 10^{12}$ protons (cyan curve), $4.5 \times 10^{12}$ protons (magenta curve), $5.2 \times 10^{12}$ protons (yellow curve).

Fig. 1(b) synchronous phase measured at extracted beam intensities of $0.315 \times 10^{12}$ protons (black curve), $1.18 \times 10^{12}$ protons (red curve), $2.1 \times 10^{12}$ protons (green curve), $2.92 \times 10^{12}$ protons (blue curve), $3.74 \times 10^{12}$ protons (cyan curve), $4.5 \times 10^{12}$ protons (magenta curve), $5.2 \times 10^{12}$ protons (yellow curve).

Fig. 1(c) synchronous phase at the period between injection at $\mathrm{t}=0$ and $8.5 \mathrm{~ms}$ for extracted beam intensities of $0.315 \times 10^{12}$ protons (black curve), $1.18 \times 10^{12}$ protons (red curve), $2.1 \times 10^{12}$ protons (green curve), $2.92 \times 10^{12}$ protons (blue curve), $3.74 \times 10^{12}$ protons (cyan curve), $4.5 \times 10^{12}$ protons (magenta curve), $5.2 \times 10^{12}$ protons (yellow curve).

Fig. 1(d) the accelerating voltage per beam turn required by $\mathrm{dB} / \mathrm{dt}$ in a Booster cycle.

Fig. 1(e) the energy loss per beam turn estimated directly from the difference between the effective accelerating voltage and the accelerating voltage required by $\mathrm{dB} / \mathrm{dt}$ at extracted beam intensities of $0.315 \times 10^{12}$ protons (black curve), $1.18 \times 10^{12}$ protons (red curve), $2.1 \times 10^{12}$ protons (green curve), $2.92 \times 10^{12}$ protons (blue curve), $3.74 \times 10^{12}$ protons (cyan curve), $4.5 \times 10^{12}$ protons (magenta curve), $5.2 \times 10^{12}$ protons (yellow curve). 


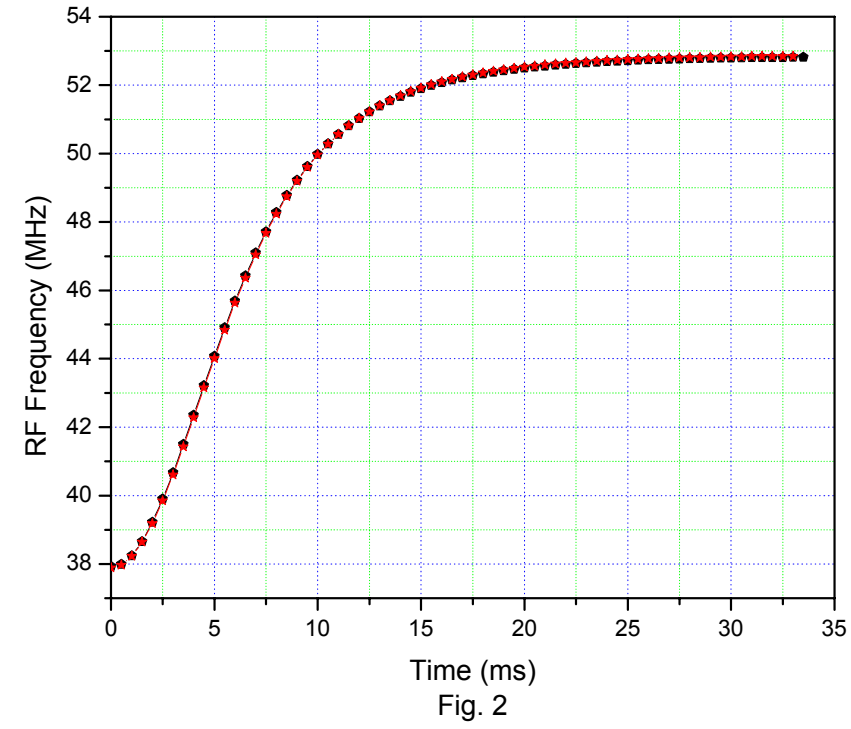

Fig. 2 the measured rf frequency in a Booster cycle at the extracted beam intensity of $5.2 \times 10^{12}$ protons is shown as the black curve, and the calculated rf frequency is shown as the red curve. 


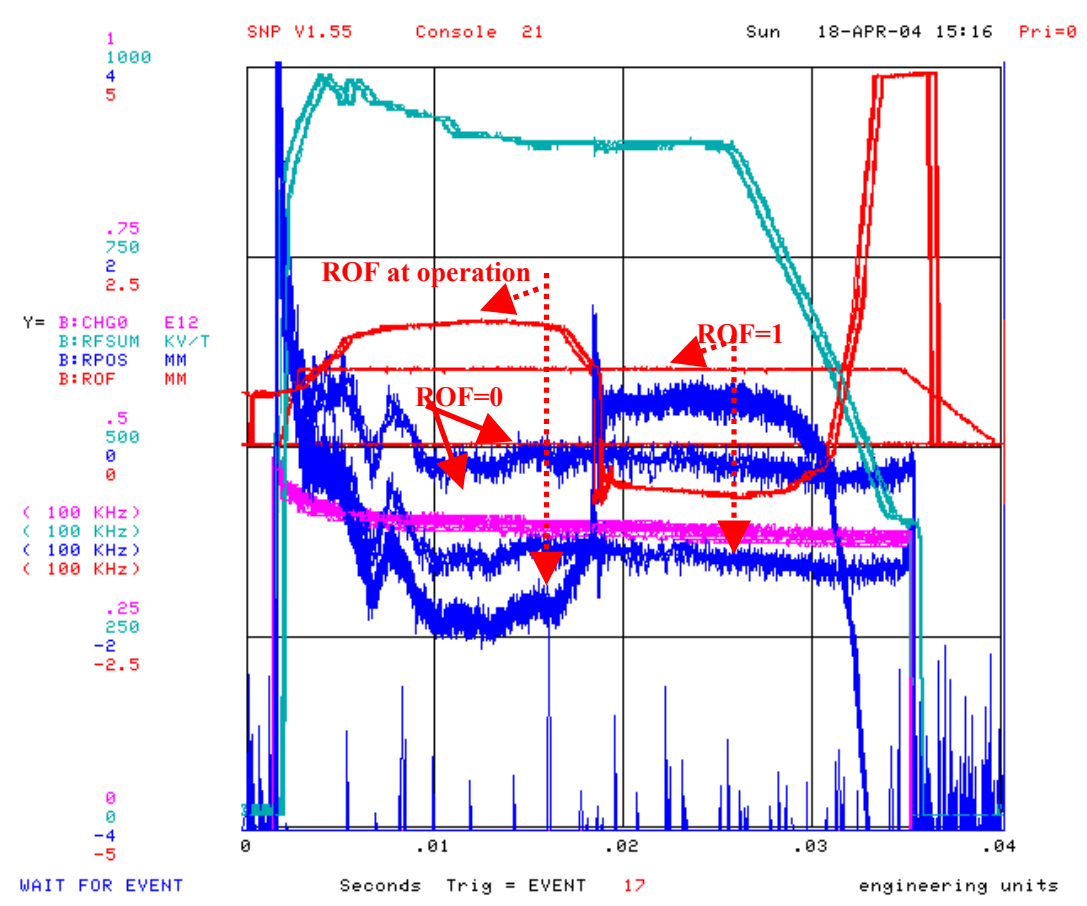

Fig. 3(a)

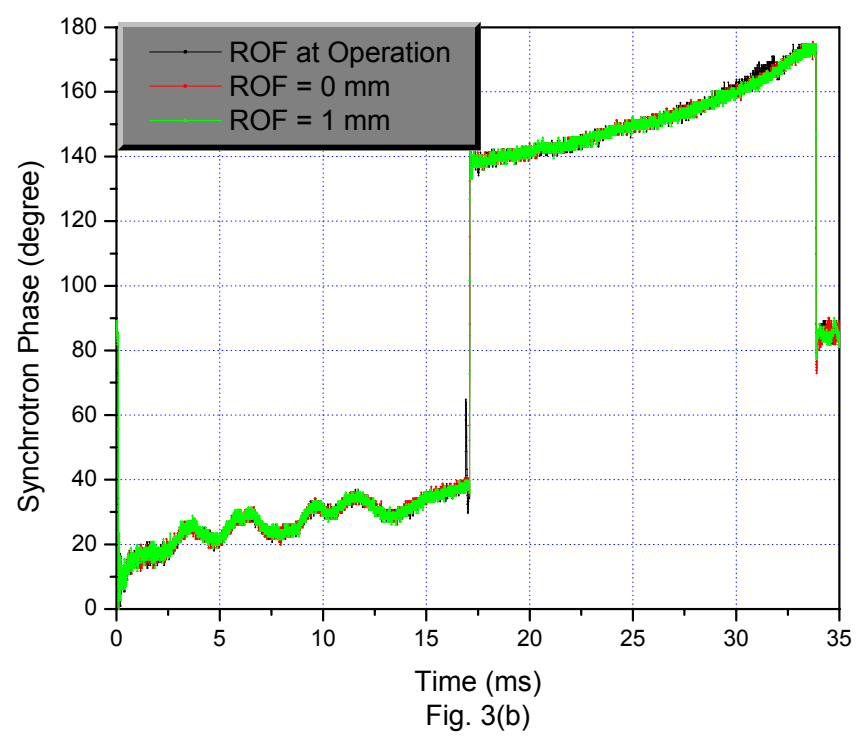

Fig. 3(a) ROF was set at 1, 0 and operational condition separately at the extracted beam intensity of $0.315 \times 10^{12}$ protons, shown as the red curves, and the corresponding radial position (RPOS) are shown as the blue curves.

Fig. 3(b) synchronous phase measured at the extracted beam intensity of $0.315 \times 10^{12}$ protons when ROF at 1 , as shown by the red curve, ROF at 0 , as shown by the green curve, and ROF at operational condition, as shown by the black curve. 


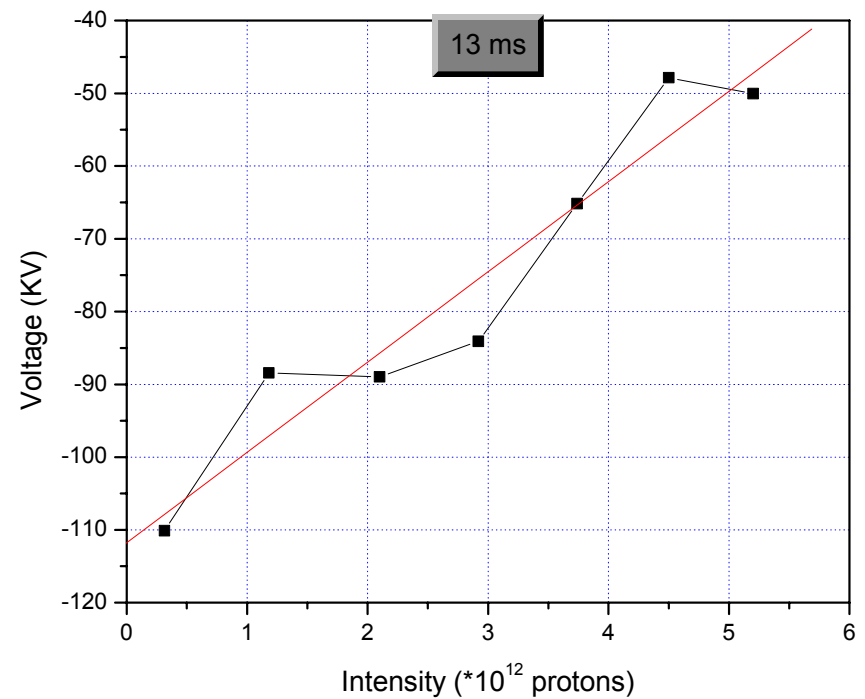

Fig. 4(a)

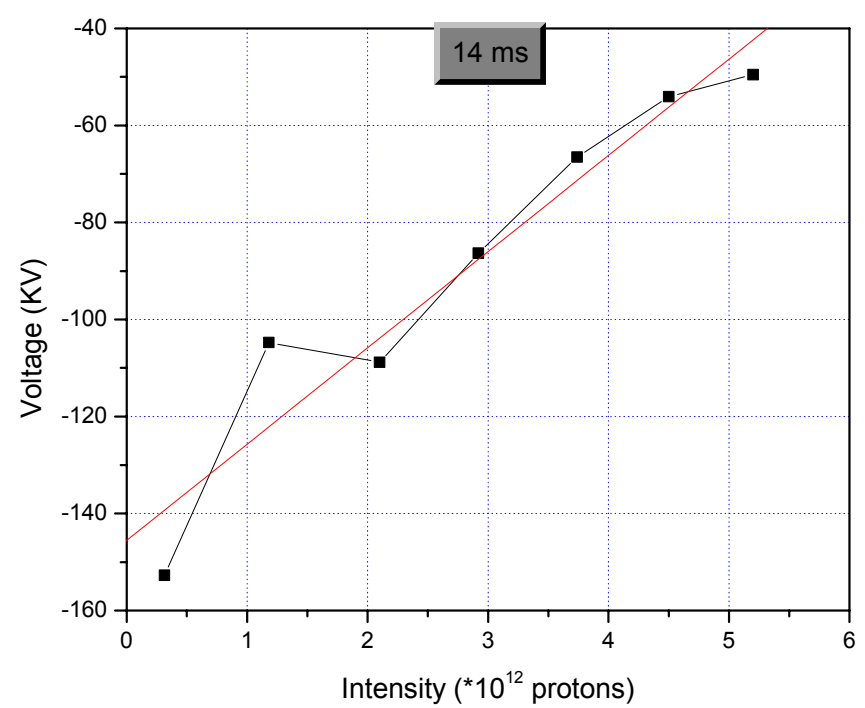

Fig. 4(b) 


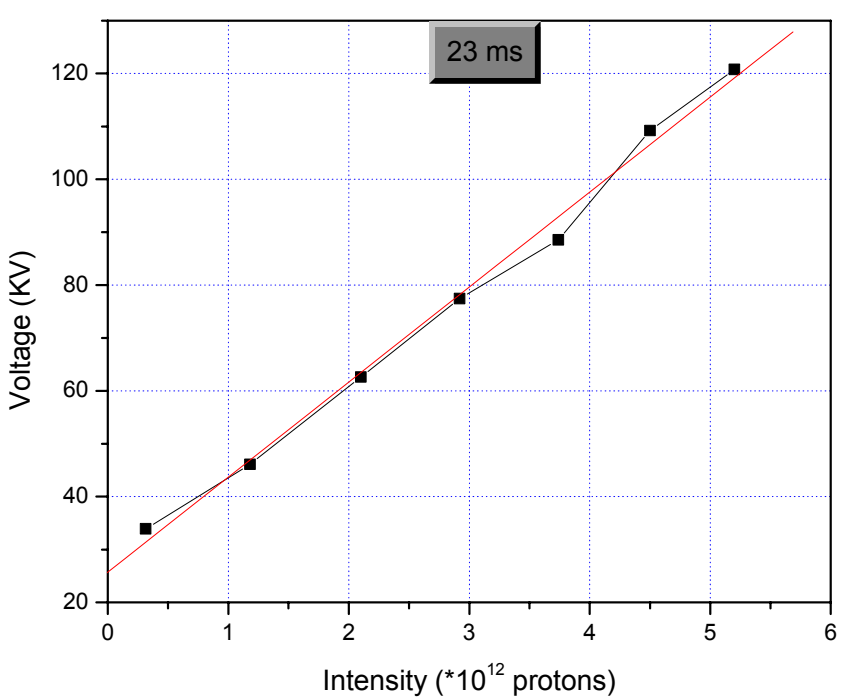

Fig. 4(c)

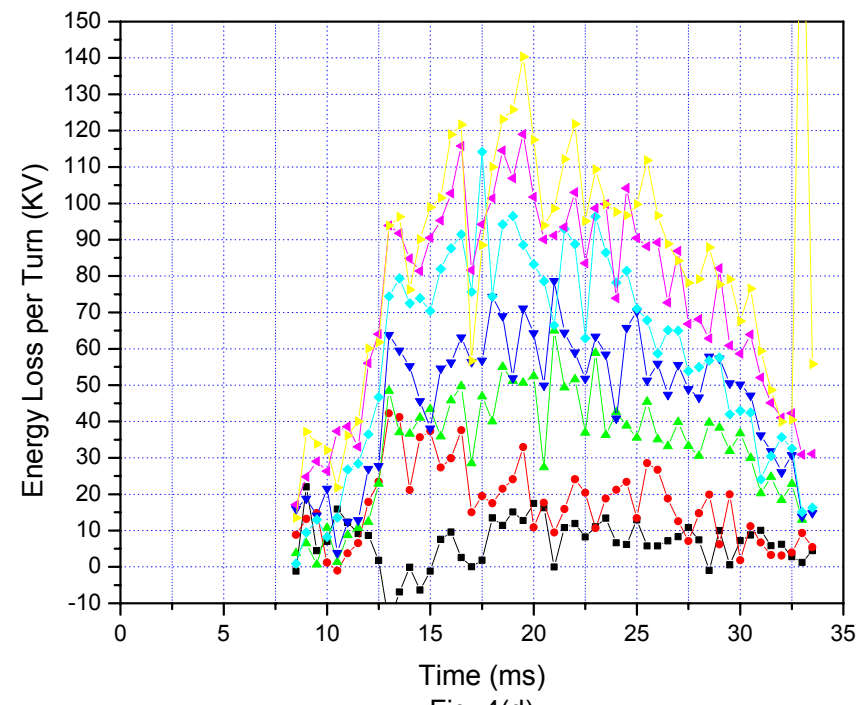

Fig. 4(d) 


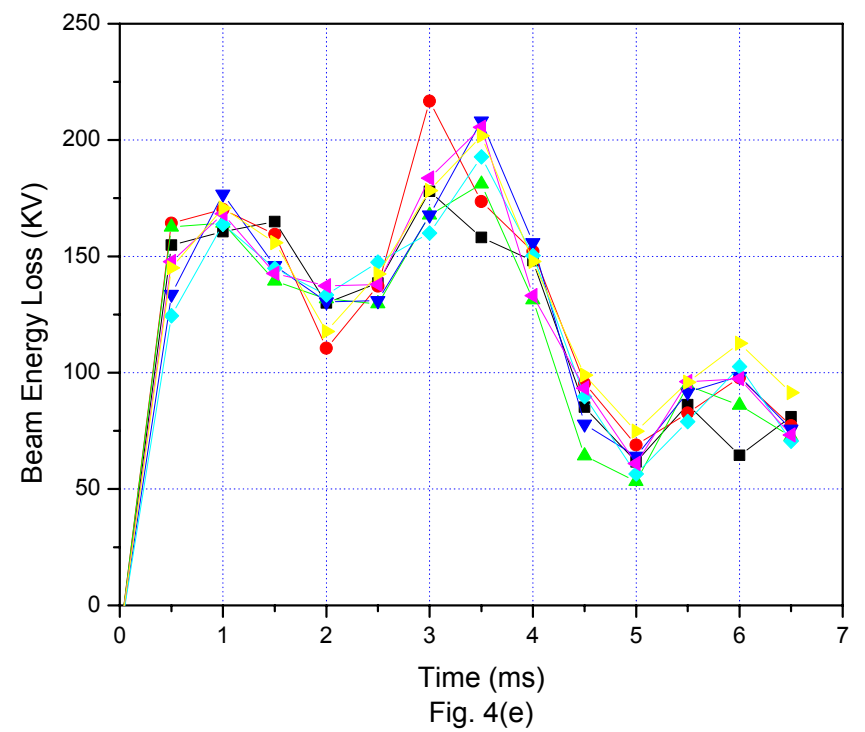

Fig. 4(a) the linear fit (red line) to the beam energy loss vs. intensity (black curve) at 13.0 ms.

Fig. 4(b) the linear fit (red line) to the beam energy loss vs. intensity (black curve) at 14.0 ms.

Fig. 4(c) the linear fit (red line) to the beam energy loss vs. intensity (black curve) at 23.0 ms.

Fig. 4(d) the beam energy loss with the correction of the offset during the period of 8.5 $\mathrm{ms}$ to extraction at extracted beam intensities of $0.315 \times 10^{12}$ protons (black curve), $1.18 \times 10^{12}$ protons (red curve), $2.1 \times 10^{12}$ protons (green curve), $2.92 \times 10^{12}$ protons (blue curve), $3.74 \times 10^{12}$ protons (cyan curve), $4.5 \times 10^{12}$ protons (magenta curve), $5.2 \times 10^{12}$ protons (yellow curve).

Fig. 4(e) the beam energy loss without the correction of the offset during the period of 0 $\mathrm{ms}$ to $6.5 \mathrm{~ms}$ at extracted beam intensities of $0.315 \times 10^{12}$ protons (black curve), $1.18 \times 10^{12}$ protons (red curve), $2.1 \times 10^{12}$ protons (green curve), $2.92 \times 10^{12}$ protons (blue curve), $3.74 \times 10^{12}$ protons (cyan curve), $4.5 \times 10^{12}$ protons (magenta curve), $5.2 \times 10^{12}$ protons (yellow curve). 